\title{
Paracoccidioidomycosis in a child: exuberant presentation due to the inadvertent use of systemic corticosteroids ${ }^{*}$
}

\author{
John Verrinder Veasey ${ }^{1}$, Nabila Scabine Pessotti ${ }^{1}$, Rute Facchini Lellis ${ }^{2}$
}

DOI: http:/ / dx.doi.org/10.1590/abd1806-4841.20198355

\begin{abstract}
Paracoccidioidomycosis is a fungal infection that occurs in immunocompetent patients and are classified into two forms: the acute-subacute form, predominantly in young patients, and the chronic adult form that may present classic ulcerated lesions to rare sarcoid ones. We present the case of a boy whose infection began with sarcoid lesions but, after being mistakenly diagnosed with cutaneous sarcoidosis and treated (for three years) with prednisone, developed painful ulcerations throughout the body. After the correct diagnosis, with evidence of the fungus in histopathological and mycological examinations, the patient was properly treated with itraconazole for eight months and evolved with total remission of the disease.
\end{abstract}

Keywords: Glucocorticoids; Child; Diagnosis; Fungi; Histology; Itraconazole; Mycoses; Paracoccidioidomycosis; Sarcoidosis; Therapeutics

The cutaneous manifestations of paracoccidioidomycosis (PCM) are classically divided into two forms: an acute-subacute juvenile form, which usually affects individuals under the age of 30 and shows a fungal tropism for the monocytic-phagocytic system, and a chronic adult form, which can be multifocal or unifocal and mild, moderate, or severe. ${ }^{1,2}$ In the chronic form, cutaneous lesions may occur as ulcers or sarcoid infiltrated plaques and can be clinically and histologically mistaken with other diseases such as leprosy, sarcoidosis, and cutaneous tuberculosis. ${ }^{1,3}$

We report a case of a 13-year-old boy who initially presented nodules, sarcoid plaques infiltrated in the face and chest, and an enlargement of the cervical lymph node. At that moment, he underwent a lymph node biopsy that revealed a granulomatous inflammatory process where necrosis was absent but numerous multinucleated giant cells, including those containing "asteroid corpuscles", were present. The diagnosis of sarcoidosis was initially proposed and the patient was treated with prednisone $20 \mathrm{mg}$ /day for three years. However, the cutaneous lesions evolved with painful ulcerations that had an unpleasant smell and a purulent secretion and were associated with a severe cervical lymph node enlargement (Figure 1). He was then referred to a reference center where other diagnostic possibilities were evaluated.

At the reference center, samples were collected from ulcers and lesions by skin scraping and skin biopsy, respectively. In the

\footnotetext{
Received 07 April 2018.

Accepted 22 September 2018.

Study conducted at the Dermatology Clinic, Santa Casa de São Paulo, São Paulo, SP, Brazil.

Financial support: None.

Conflict of interest: None.

Dermatology Clinic, Santa Casa de São Paulo - São Paulo, SP, Brazil.

Laboratory of Pathology, Santa Casa de São Paulo - São Paulo, SP, Brazil.

\section{MAILING AdDRESS:}

John Verrinder Veasey

E-mail: johnveasey@uol.com.br
} 


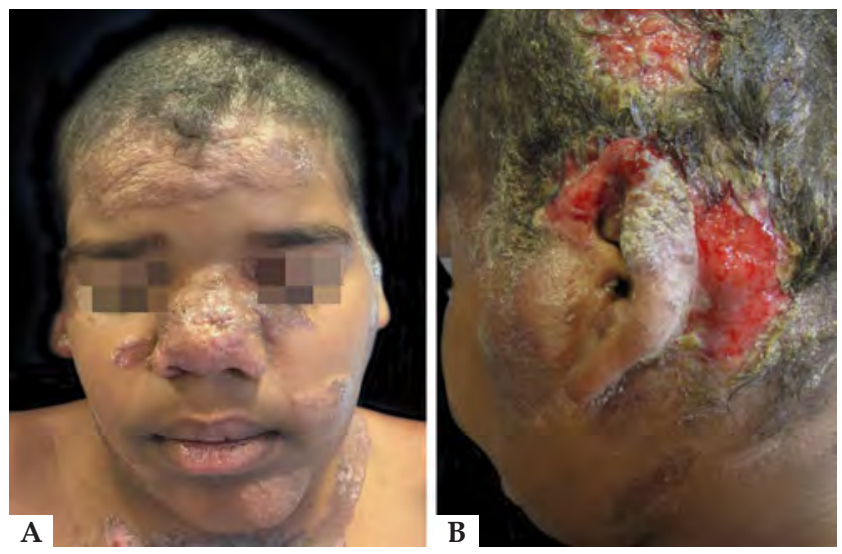

Figure 1: Clinical aspect of the lesions. A - Infiltrated erythematous plaques with a sarcoid aspect on the earlobes and on the frontal, nasal, bilateral malar, and cervical regions. B - Intense edema in the pre-auricular region and left auricle, with ulceration extending to the retroauricular region, and synechia formation. Another infiltrated and ulcerated plaque in the left temporal region is noted
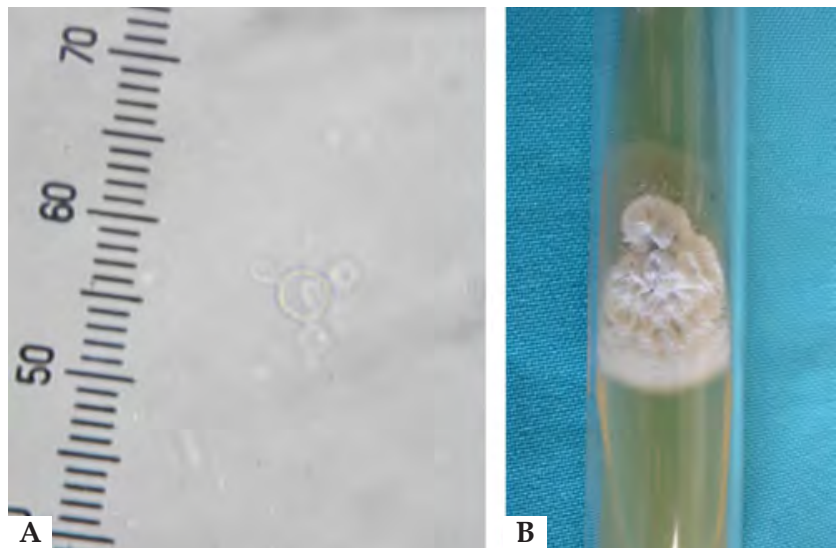

Figure 2: Mycological examinations of paracoccidioidomycosis. A. Direct mycological observation through optical microscopy (X200) with $10 \% \mathrm{KOH}$, showing birefringent yeasts with multiple buds. B. Fungal culture in Sabouraud medium showing the growth of a filamentous colony with a rough aspect at $25^{\circ} \mathrm{C}$ direct mycological examination, the presence of isolated and rare yeasts with multiple buds, suggestive of Paracoccidioides sp. (Figure 2), was observed. Histopathological examination revealed a histiocytic reaction with epithelioid granulomas and multinucleated giant cells that contained multiple bud-yeast structures (Figure 3). Fungal cultures of the samples collected from ulcers and lesions presented growth of Paracoccidioides sp. (Figure 2). A chest X-ray showed no pulmonary involvement.

The patient was hospitalized for treatment with itraconazole $400 \mathrm{mg} /$ day. Additionally, the patient was treated for a secondary bacterial infection with vancomycin and ceftazidime for ten days. Itraconazole was reduced to $200 \mathrm{mg}$ / day and maintained for eight months. The patient evolved with a gradual improvement, during which only scarring lesions could be observed (Figure 4). After one year and three months of follow-up, the patient did not show re-occurring lesions.

PCM with sarcoid manifestation usually occurs in young patients who may present obstructed cervical lymph nodes but a good general condition, similar to the case initially reported. ${ }^{1,4}$ Fre-
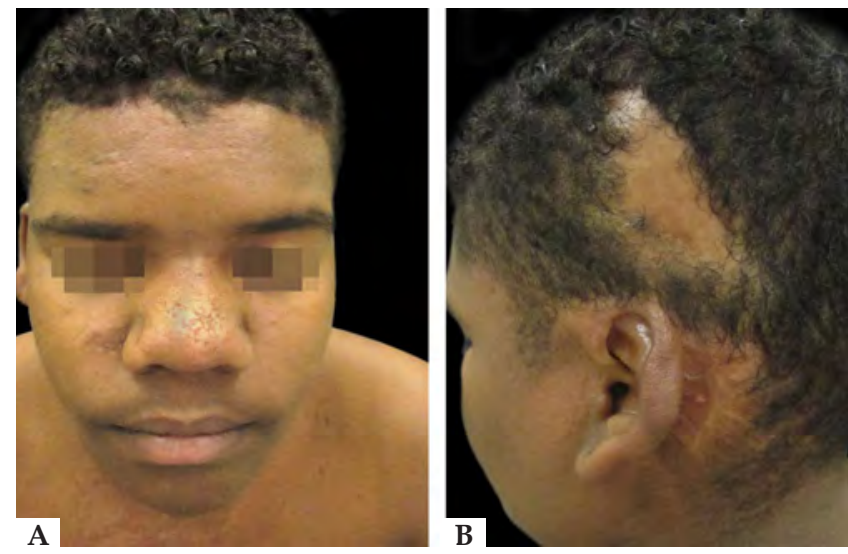

Figure 4: Clinical aspect of the lesions after eight months of treatment showing scaring lesions
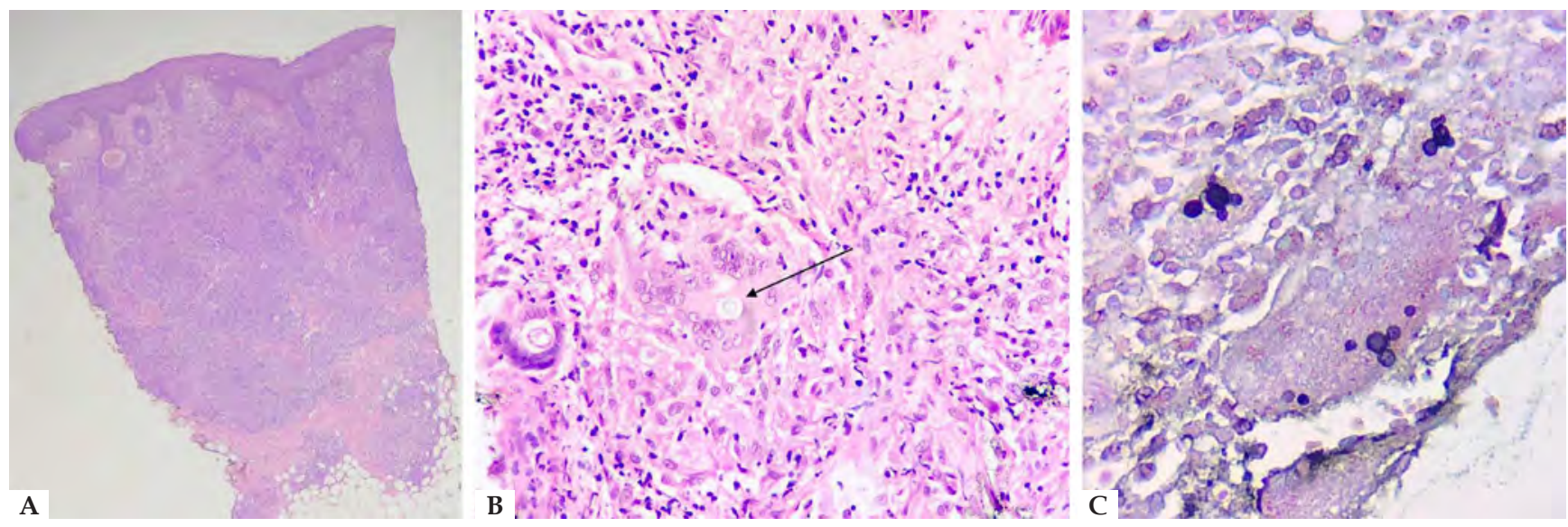

Figure 3: A. The histological section revealed a granulomatous nodular dermatosis compromising the entire dermis. Few portions of the epidermis were acanthotic and no pseudoepitheliomatous hyperplasia was observed (Hematoxylin \& eosin, x25). B. A yeast with multiple buds is observed within the granuloma (arrow) (Hematoxylin \& eosin, x400). C. Yeasts with multiple buds in the granuloma (Grocott stain, x400) 
quently, this manifestation is reported as being caused by misdiagnosis, justifying the initial diagnosis of sarcoidosis in this case. ${ }^{4-6}$ The sarcoid pattern of PCM can be determined by a specific immune response, which leads to a certain balance between agent and host and generates paucifungal histological exams, hindering the correct diagnosis. ${ }^{7}$ In this case, treatment with prednisone probably altered such balance, favoring an immunosuppression that allowed lesions to evolve into ulcerated forms, which are more typical of the disease and contain an increased amount of yeasts, facilitating the correct diagnosis. Thus, this case illustrates the importance of isolating the causative agent in granulomatous diseases that are clinically and histopathologically similar to the sarcoid pattern.]

\section{REFERENCES}

1. Marques SA. Paracoccidioidomycosis: epidemiological, clinical, diagnostic and treatment up-dating. An Bras Dermatol. 2013;88:70112.

2. Shikanai-Yasuda MA, Mendes RP, Colombo AL, Queiroz-Telles F, Kono ASG, Paniago AMM, et al. Brazilian guidelines for the clinical management of paracoccidioidomycosis. Rev Soc Bras Med Trop. 2017;50:715-740.

3. Martinez R. Epidemiology of paracoccidioidomycosis. Rev Inst Med Trop Sao Paulo. 2015;57(Suppl 19):11-20.

4. Marques SA, Lastória JC, Putinatti MS, Camargo RM, Marques ME. Paracoccidioidomycosis: infiltrated, sarcoid-like cutaneous lesions misinterpreted as tuberculoid leprosy. Rev Inst Med Trop Sao Paulo. 2008;50:47-50.
5. Fernandes NC, Côrtes JG, Akitti T, Quintella DC, Cuzzi T. Sarcoid-like cutaneous lesions in chronic adult paracoccidioidomycosis: report of two cases. Rev Inst Med Trop Sao Paulo. 2017;59:e36.

6. Nascimento $\mathrm{CR}$, Delanina WF, Soares $\mathrm{CT}$.. Paracoccidioidomycosis: sarcoid-like form in childhood. An Bras Dermatol. 2012;87:486-7.

7. Medeiros VLS, Arruda L. Sarcoid-like lesions in Paracoccidioidomycosis: immunological factors. An Bras Dermatol. 2013; 88:113-6.

\footnotetext{
AUTHORS' CONTRIBUTIONS

John Verrinder Veasey $\quad$ (D) ORCID 0000-0002-4256-5734

Approval of the final version of the manuscript; Conception and planning of the study; Elaboration and writing of the manuscript; Obtaining, analyzing and interpreting the data; Effective participation in research orientation; Intellectual participation in propaedeutic and/or therapeutic conduct of the cases studied; Critical review of the literature; Critical review of the manuscript.

Nabila Scabine Pessotti $\quad$ (D) ORCID 0000-0003-0879-2981

Elaboration and writing of the manuscript; Obtaining, analyzing and interpreting the data; Critical review of the literature; Critical review of the manuscript.

Rute Facchini Lellis $\quad$ (D) ORCID 0000-0001-7690-0513

Conception and planning of the study; Obtaining, analyzing and interpreting the data; Intellectual participation in propaedeutic and/or therapeutic conduct of the cases studied.
}

How to cite this article: Veasey JV, Pessotti NS, Lellis RF. Paracoccidioidomycosis in a child: exuberant presentation due to the inadvertent use of systemic corticosteroids. . An Bras Dermatol. 2019;94(4):470-2. 